\title{
Early Decline in Left Ventricular Ejection Fraction Can Predict Trastuzumab-Related Cardiotoxicity in Patients with Breast Cancer: A Study Using 13 Years of Registry Data
}

\author{
Eun Kyoung Kim, MD, PhD' \\ Jinhyun Cho, MD² \\ Ji-Yeon Kim, $\mathrm{MD}^{3}$ \\ Sung-A Chang, MD, PhD 1 \\ Sung-Ji Park, MD, $P D^{1}$ \\ Jin Oh Choi, MD, PhD \\ Sang Chol Lee, MD, PhD \\ Jin Seok Ahn, MD, PhD ${ }^{3}$ \\ Seung Woo Park, MD, PhD' \\ Young-Hyuck Im, MD, PhD 3 \\ Eun Seok Jeon, MD, PhD ${ }^{1}$ \\ Yeon Hee Park, MD, PhD ${ }^{3}$
}

${ }^{1}$ Division of Cardiology, Heart Vascular Stroke Institute, Samsung Medical Center, Sungkyunkwan University School of Medicine, Seoul, ${ }^{2}$ Division of Hematology-Oncology, Department of Medicine, Inha University Hospital, Inha University School of Medicine, Incheon, ${ }^{3}$ Division of Hematology-Oncology, Department of Medicine, Samsung Medical Center, Sungkyunkwan University School of Medicine, Seoul, Korea
Correspondence: Yeon Hee Park, MD, PhD Division of Hematology-Oncology, Department of Medicine, Samsung Medical Center, Sungkyunkwan University School of Medicine, 81 Irwon-ro, Gangnam-gu, Seoul 06351, Korea

Tel: 82-2-3410-3459

Fax: 82-2-3410-1754

E-mail: yeonh.park@samsung.com

Received May 4, 2018

Accepted September 3, 2018

Published Online September 4, 2018

*Eun Kyoung Kim and Jinhyun Cho contributed equally to this work.

\section{Purpose}

While concerns regarding trastuzumab-related cardiac dysfunction (TRCD) in patients with breast cancer are increasing, there is a lack of evidence supporting the current recommendations for TRCD monitoring. We aimed to investigate the clinical predictors of TRCD in the adjuvant setting of human epidermal growth factor receptor 2-positive breast cancer patients.

\section{Materials and Methods}

From August 2003 to April 2016, consecutive 998 patients who were treated with adjuvant trastuzumab for breast cancer were retrospectively evaluated. TRCD was defined as a decrease $\geq 10 \%$ in left ventricular ejection fraction (LVEF), with a decline below the normal limit or symptomatic heart failure.

\section{Results}

Among 787 eligible patients who had complete data sets consisting of both baseline and follow-up assessment of left ventricular systolic function by echocardiography (mean age, 49.9 \pm 9.5 years), 58 (7.4\%) developed TRCD. TRCD patients had lower baseline LVEF (63\% [59-66] vs. $65 \%$ [61-68], $p=0.016$ ) and more frequently administered Adriamycin ( $98 \%$ vs. $89 \%, p=0.022$ ) than those without TRCD. On follow-up echocardiography, a drop in LVEF $\geq 5 \%$ within the first 3 months was more frequent in TRCD patients $(78.3 \%$ vs. $38.4 \%, p<$ 0.001). Regardless of baseline LVEF and adriamycin treatment, a drop in LVEF $\geq 5 \%$ within the first 3 months of trastuzumab administration was strongly associated with the development of TRCD (adjusted hazard ratio, 45.1 [17.0-127.6]; $p<0.001$ ).

\section{Conclusion}

The overall incidence of TRCD was $7.4 \%$ in Asian breast cancer patients treated with adjuvant trastuzumab. A decline in LVEF $\geq 5 \%$ within the first 3 months of trastuzumab initiation was strongly associated with TRCD development in patients with breast cancer.

\section{Key words}

Breast neoplasms, Trastuzumab, Cardiotoxicity, Left ventricular ejection fraction 


\section{Introduction}

Trastuzumab (Herceptin, Genentech Inc.), a humanized monoclonal antibody against the extracellular domain of the human epidermal growth factor receptor 2 (HER2), is part of the standard adjuvant therapy for HER2-positive breast cancer [1]. While trastuzumab has yielded considerable improvement in cancer outcomes in these patients, its potential cardiotoxicity is a major concern. The reported incidence of trastuzumab-related cardiac dysfunction (TRCD) varies according to the definition and its severity, which ranges from $7 \%-34 \%$ for asymptomatic left ventricular (LV) systolic dysfunction to $0 \%-4.1 \%$ for overt congestive heart failure $[2,3]$. Since there is currently no preventive medication for $\mathrm{TRCD}$, and discontinuation of trastuzumab frequently restores cardiac function, early detection of cardiotoxicity through close monitoring during the use of trastuzumab has been the only therapeutic option in HER2-positive breast cancer patients $[4,5]$

Some researchers have reported the presence of hypertension, smoking history, advanced age and coronary artery disease as risk factors for the development of TRCD and have emphasized that patients with those preexisting cardiac risk factors should be monitored more intensively during trastuzumab administration $[1,6,7]$. However, the risk stratification of patients who warrant close cardiac surveillance or the institution of a cardioprotective strategy is inconsistent and differs between studies or between races. In Asian patients with breast cancer, in particular, little is known about clinical manifestations and predictors of TRCD.

Although some guidelines have proposed a monitoring strategy for TRCD using left ventricular ejection fraction (LVEF), which focuses mainly on baseline LV function, they are largely based on expert opinion and lack evidence to support their effectiveness $[8,9]$. While lower baseline LVEF is an objective parameter for the prediction of cardiotoxicity in patients who are treated with trastuzumab, it has limited negative predictive value [10]. Moreover, the development of TRCD in patients with normal LVEF was reported in previous clinical trials [11,12]. Therefore, serial changes in LVEF may be more important as a parameter for detecting cardiac dysfunction. Overall, there is a growing need for data to support current guidelines regarding the interval and threshold value of examinations for the early detection of TRCD. In the present study, we aimed to investigate the incidence and clinical manifestations of TRCD and to identify its predictors in the setting of adjuvant chemotherapy for HER2-positive breast cancer.

\section{Materials and Methods}

\section{Study population and design}

The study cohort consisted of consecutive patients who were treated with trastuzumab in the adjuvant setting of HER2-positive breast cancer between August 2003 and April 2016 and who had echocardiography data before the administration of trastuzumab and during follow-up within the medication schedule. All patients were diagnosed with histologically confirmed, completely excised invasive breast cancer with HER2-positive, fulfilling the institutional criteria for adjuvant therapy with trastuzumab. Among 998 eligible patients, we excluded patients who discontinued trastuzumab in the early period $(n=38)$ and did not undergo fol-

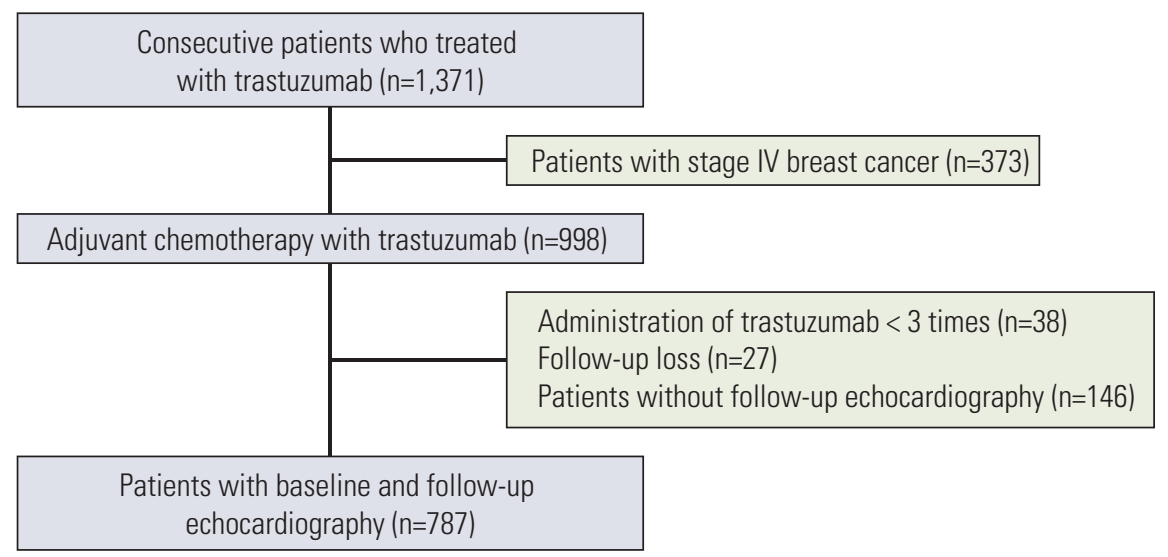

Fig. 1. Study design and population. 
low-up echocardiography $(\mathrm{n}=146)$. After the exemption of 27 patients who were lost to follow-up, a total of 787 patients were included in final analysis (Fig. 1). Trastuzumab was administered as a single loading dose of $8 \mathrm{mg} / \mathrm{kg}$ of body weight intravenously, followed by maintenance doses of 6 $\mathrm{mg} / \mathrm{kg}$ every 3 weeks for 1 year. Patients were routinely followed after every cycle of trastuzumab and annually thereafter. Clinical characteristics and adverse cardiac event data were collected from our breast cancer registry by research coordinators. Further information was collected by extensive review of medical records. The median follow-up duration was 54.3 months (range, 37.3 to 75.1 months).

Comprehensive transthoracic echocardiography (Vivid 7, GE Medical Systems, Milwaukee, WI; Acuson 512, Siemens Medical Solution, Mountain View, CA; or Sonos 5500, Philips Medical System, Andover, MA) was performed in all enrolled patients using established guidelines for the evaluation of LV systolic function [13]. LVEF was assessed via biplane Simpson's rule using manual tracing of digital images from the apical view. The same echocardiographic vendor was used to monitor change of LVEF in each patient. During trastuzumab treatment, all patients underwent echocardiography at baseline (before administration of trastuzumab) and every 3 months to monitor cardiac function.

Trastuzumab-mediated cardiotoxicity was defined according to the criteria established by clinical trials and current guidelines: (1) a decline in LVEF of at least 10\% below 50\% without accompanying signs or symptoms of heart failure; or (2) symptomatic heart failure, necessitating discontinuation of the drug $[1,8,9,14,15]$. Trastuzumab treatment was stopped and referral to a cardiologist was made when patients developed heart failure or when LVEF dropped below $45 \%$ regardless of heart failure symptoms. The cardiologist's intervention was performed within 1 week after the LV dysfunction was detected. Cardioprotective medications including carvedilol and angiotensin-converting enzyme inhibitor / angiotensin receptor blocker were titrated according to the patient's blood pressure and heart rate. In patients who developed TRCD, New York Heart Association (NYHA) functional class, cardioprotective medication, whether the dysfunction was reversible, and whether trastuzumab was continued were assessed. The reversible TRCD was defined as if the LVEF was recovered to the baseline.

\section{Statistical analysis}

Baseline characteristics were summarized with continuous variables and expressed as mean \pm standard deviation or median with interquartile range. Categorical data are presented as a percentage and the number of events. Continuous variables were analyzed using an independent $t$ test or Wilcoxon rank sum test as appropriate, and categorical vari- ables were analyzed using a chi-square test or Fisher exact test. Univariate and multivariate time dependent Cox regression analysis were performed to identify baseline characteristics that were independently associated with TRCD. We included in multivariate models those covariates that were significant on univariate analysis $(\mathrm{p}<0.2)$ and those that were clinically relevant. We additionally performed receiver operating characteristic curve analysis to identify the LVEF cut-off value for discriminating patients at high risk of TRCD from the low-risk group. All analysis was conducted with SPSS software ver. 17.0 (SPSS Inc., Chicago, IL). Statistical significance was concluded at a 2-sided significance level of 0.05 for all analyses.

\section{Ethical statement}

The local institutional review board approved this study (Samsung Medical Center IRB No. 2017-12-078) and waived the requirement for written informed consent for access to institutional breast cancer registry data.

\section{Results}

\section{Baseline characteristics}

A total of 787 patients with breast cancer who were treated with trastuzumab in an adjuvant setting and had complete data sets consisting of both baseline and follow-up assessment of LV systolic function by echocardiography were analyzed (age, $49.9 \pm 9.5$ years). During the follow-up period, TRCD developed in 58 patients $(7.4 \%)$ who presented with asymptomatic $\mathrm{LV}$ systolic dysfunction $(\mathrm{n}=40,5.1 \%)$ or symptomatic heart failure $(n=18,2.3 \%)$. Table 1 summarizes the baseline clinical characteristics of the patients. All patients were NYHA functional class I at baseline. Preexisting histories of known cardiovascular risk factors such as hypertension, diabetes, dyslipidemia, smoking and coronary artery disease did not differ between patients with and without TRCD (all $p>0.05$ ). Cardiac medication profiles at the presentation of TRCD did not differ between the two groups. Patients in whom TRCD developed had higher initial blood pressure compared to those without TRCD (systolic blood pressure, 125 [107-136] mmHg vs. 114 [104-126] mmHg; $\mathrm{p}=0.005$ and diastolic blood pressure, 72 [65-81] $\mathrm{mmHg}$ vs. 68 [61-76] mmHg; $\mathrm{p}=0.004)$. On electrocardiography, most enrolled patients had normal sinus rhythm, except a few patients with atrial fibrillation or frequent ventricular premature beat, which were not significantly different between the two groups. Baseline LVEF was lower in patients with 
Table 1. Comparison between patients with and without TRCD

\begin{tabular}{|c|c|c|c|}
\hline Variable & $\begin{array}{c}\text { No TRCD } \\
(n=729)\end{array}$ & $\begin{array}{l}\text { TRCD } \\
(n=58)\end{array}$ & p-value \\
\hline Ethnicity, Asian & $729(100)$ & $58(100)$ & - \\
\hline Age (yr) & $50(43-57)$ & $49(45-54)$ & 0.348 \\
\hline Weight (kg) & $58(52.6-64.0)$ & $57.0(52.8-60.3)$ & 0.371 \\
\hline BMI $\left(\mathrm{kg} / \mathrm{m}^{2}\right)$ & $23.2(21.2-25.7)$ & $23.0(21.8-24.7)$ & 0.768 \\
\hline Obesity & $226(31.0)$ & $11(19.0)$ & 0.054 \\
\hline Hypertension & $124(17.0)$ & $6(10.3)$ & 0.188 \\
\hline Diabetes & $35(4.8)$ & $3(5.2)$ & 0.754 \\
\hline Dyslipidemia & $43(5.9)$ & $2(3.4)$ & 0.766 \\
\hline Previous CAD & $4(0.5)$ & $1(1.7)$ & 0.319 \\
\hline \multicolumn{4}{|l|}{ Smoking } \\
\hline Current & $6(0.8)$ & $1(1.7)$ & 0.537 \\
\hline Ex-smoker & $3(0.4)$ & - & \\
\hline Cerebrovascular disease & $14(1.9)$ & - & 0.615 \\
\hline \multicolumn{4}{|l|}{ Current medication } \\
\hline $\mathrm{ARB} / \mathrm{ACEi}$ & $52(7.1)$ & $3(5.2)$ & 0.790 \\
\hline Beta-blocker & $14(1.9)$ & $1(1.7)$ & 1.000 \\
\hline Statin & $36(4.9)$ & $2(3.4)$ & 1.000 \\
\hline Diuretics & $31(4.3)$ & $2(3.4)$ & 1.000 \\
\hline Systolic blood pressure (mmHg) & $114(104-126)$ & $125(107-136)$ & 0.005 \\
\hline Diastolic blood pressure (mmHg) & $68(61-76)$ & $72(65-81)$ & 0.004 \\
\hline Heart rate (beats/min) & $72(66-80)$ & $73(67-86)$ & 0.207 \\
\hline \multicolumn{4}{|l|}{ Laboratory findings } \\
\hline GFR (mL / min) & $43.2(35.9-56.3)$ & $41.1(35.5-50.4)$ & 0.227 \\
\hline Hemoglobin (g/dL) & $11.9(11.1-12.6)$ & $11.7(11.2-12.7)$ & 0.695 \\
\hline Total cholesterol (mg/dL) & $202(178-227)$ & $207.5(187.8-229.3)$ & 0.141 \\
\hline \multicolumn{4}{|l|}{ Electrocardiographic finding } \\
\hline Atrial fibrillation & $4(0.5)$ & - & 1.000 \\
\hline Frequent VPCs & $12(1.6)$ & $2(3.4)$ & 0.276 \\
\hline \multicolumn{4}{|l|}{ Echocardiographic findings } \\
\hline Pre-trasutzumab LVEF & $65.0(61.0-68.0)$ & $62.5(59.0-66.0)$ & 0.016 \\
\hline
\end{tabular}

Values are presented as number (\%) or median (range). TRCD, trastuzumab-related cardiac dysfunction; BMI, body mass index; $\mathrm{CAD}$, coronary artery disease; $\mathrm{ARB}$, angiotensin receptor blocker; $\mathrm{ACEi}$, angiotensin-converting enzyme inhibitor; GFR, glomerular filtration rate; VPC, ventricular premature beat; LVEF, left ventricular ejection fraction.

TRCD (62.5\% [59.0-66.0] vs. 65.0\% [61.0-68.0], p=0.016), but it was still within normal range. There were no significant differences in cancer treatment between the two groups including excision type and radiotherapy, excepting the use of anthracycline (Table 2). Patients with TRCD were more likely to receive anthracycline before administration of trastuzumab (98.3\% vs. $88.6 \%$, p=0.022). Anthracycline dose was significantly higher in patients who experienced TRCD than those without TRCD $\left(261.4\right.$ [237.7-294.4] $\mathrm{mg} / \mathrm{m}^{2}$ vs. 236.9 [218.1-245.9] $\left.\mathrm{mg} / \mathrm{m}^{2}, \mathrm{p}<0.001\right)$.

\section{Detection of TRCD}

In patients who experienced TRCD, the median time to development of TRCD was $6.0 \pm 2.9$ months, which is at the 10.2 \pm 5.1 times of trastuzumab administration. Baseline LVEF of $54.5 \%$ showed a significant predictive value for TRCD in receiver operating character curve analysis (area under the curve, 0.60 [0.52-0.67]; $\mathrm{p}=0.016$ ) with $98.3 \%$ sensitivity and 98.9\% specificity. Fig. 2 shows serial changes of LVEF in patients with and without TRCD. On follow-up echocardiography, a drop in LVEF at 6 months was more prominent in TRCD patients than those without TRCD (11.0\% [5.0-19.0] vs. 3.0\% [0-6.0], p < 0.001). Notably, there was also a progres- 
Table 2. Cancer presentation and treatment

\begin{tabular}{|c|c|c|c|}
\hline & $\begin{array}{c}\text { No TRCD } \\
(n=729)\end{array}$ & $\begin{array}{l}\text { TRCD } \\
(n=58)\end{array}$ & p-value \\
\hline \multicolumn{4}{|l|}{ Cancer status } \\
\hline \multicolumn{4}{|l|}{ Location } \\
\hline Right & $348(47.7)$ & $28(48.3)$ & 0.944 \\
\hline Left & $372(51.0)$ & $29(50.0)$ & \\
\hline Bilateral & $9(1.2)$ & $1(1.7)$ & \\
\hline \multicolumn{4}{|l|}{ Stage } \\
\hline 1 & $19(26.2)$ & $21(36.2)$ & 0.249 \\
\hline 2 & $360(49.4)$ & $25(43.1)$ & \\
\hline 3 & $157(21.5)$ & $12(20.7)$ & \\
\hline \multicolumn{4}{|l|}{ Treatment } \\
\hline \multicolumn{4}{|l|}{ Surgery } \\
\hline Total & $284(39.0)$ & $26(44.8)$ & 0.379 \\
\hline Partial & $445(61.0)$ & $32(55.2)$ & \\
\hline Radiotherapy & $559(76.7)$ & $40(69.0)$ & 0.185 \\
\hline Dose (cGy) & $5,900(5,900-6,050)$ & $5,900(5,900-6,050)$ & 0.940 \\
\hline Fraction & $28(28-28)$ & $28(28-28)$ & 0.085 \\
\hline Adriamycin & $646(88.6)$ & $57(98.3)$ & 0.022 \\
\hline Adriamycin dose $\left(\mathrm{mg} / \mathrm{m}^{2}\right)$ & $236.9(218.1-245.9)$ & $261.4(237.7-294.4)$ & $<0.001$ \\
\hline Epirubicin & $12(1.6)$ & $1(1.7)$ & $>0.999$ \\
\hline
\end{tabular}

Values are presented as number $(\%)$ or median (range). TRCD, trastuzumab-related cardiac dysfunction.

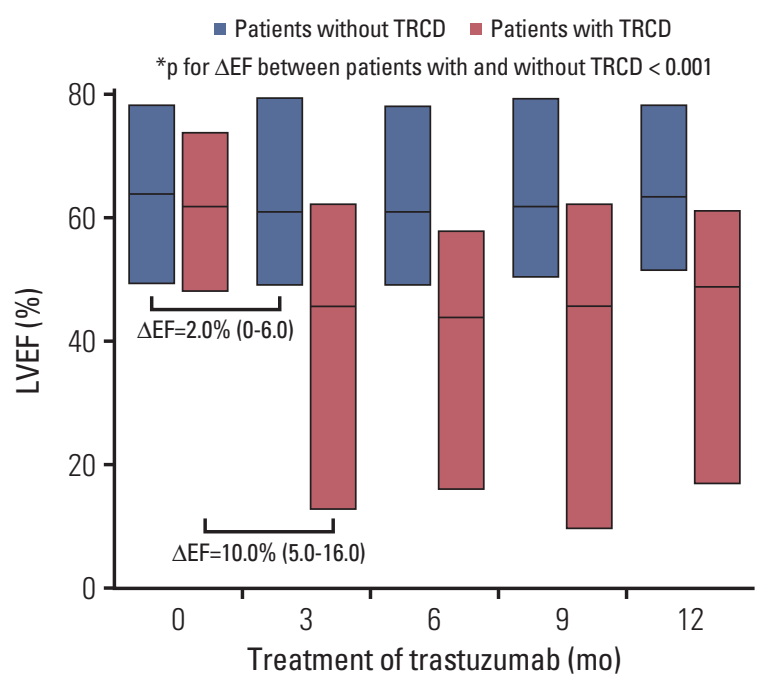

$\begin{array}{lllll}\text { No. of patients } & 787 & 787 & 514 & 363\end{array}$

Fig. 2. Serial changes in patients with and without trastuzumab-related cardiac dysfunction (TRCD). Patients with TRCD experienced significant left ventricular ejection fraction (LVEF) decrement compared to those without TRCD within the first 3 months of trastuzumab administration $(\mathrm{p}<0.001)$.

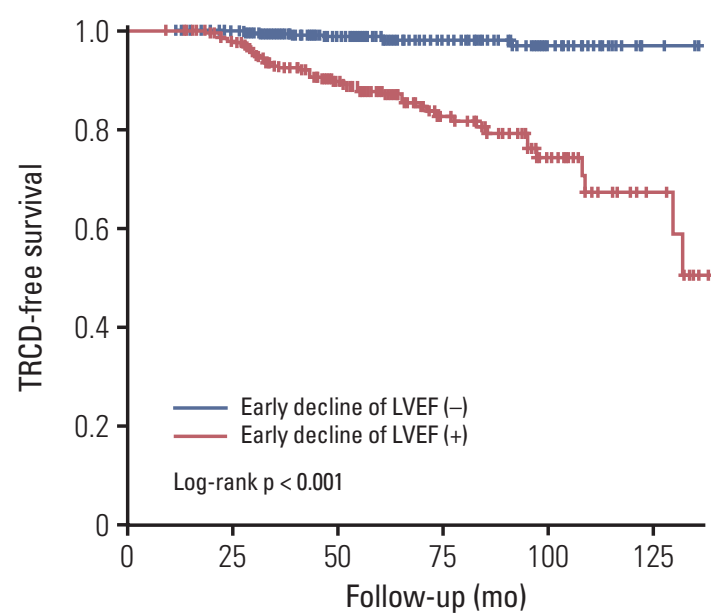

No. of patients

$\begin{array}{rcccccc}\begin{array}{r}\text { Early decline } \\ \text { of LVEF (-) }\end{array} & 449 & 430 & 238 & 115 & 40 & 3 \\ \begin{array}{r}\text { Early decline } \\ \text { of LVEF }(+)\end{array} & 337 & 317 & 196 & 81 & 37 & 9\end{array}$

Fig. 3. Kaplan-Meier curve for trastuzumab-related cardiac dysfunction (TRCD) -free survival. LVEF, left ventricular ejection fraction. 
Table 3. Univariate and multivariate time dependent Cox regression analysis for development of TRCD

\begin{tabular}{|c|c|c|c|c|}
\hline Variable & $\begin{array}{l}\text { Univariate HR } \\
\text { (95\% CI) }\end{array}$ & p-value & $\begin{array}{c}\text { Multivariate HR } \\
(95 \% \mathrm{CI})\end{array}$ & p-value \\
\hline Hypertension & $0.62(0.27-1.45)$ & 0.274 & $0.69(0.27-1.77)$ & 0.440 \\
\hline Diabetes & $0.88(0.28-2.82)$ & 0.832 & $1.13(0.32-3.98)$ & 0.852 \\
\hline CAD & $2.98(0.41-21.58)$ & 0.279 & $3.60(0.45-28.58)$ & 0.225 \\
\hline Dyslipidemia & $0.73(0.18-2.98)$ & 0.657 & $1.36(0.29-6.36)$ & 0.699 \\
\hline Smoking & $2.64(0.36-19.25)$ & 0.339 & $3.83(0.50-29.11)$ & 0.195 \\
\hline Baseline LVEF & $0.94(0.89-0.99)$ & 0.019 & $0.80(0.75-0.85)$ & $<0.001$ \\
\hline Baseline DBP & $1.03(1.00-1.05)$ & 0.052 & $1.05(1.02-1.08)$ & 0.002 \\
\hline Obesity & $0.58(0.30-1.12)$ & 0.104 & $0.43(0.21-0.86)$ & 0.018 \\
\hline Adriamycin dose & $1.01(1.00-1.01)$ & $<0.001$ & $1.01(1.00-1.01)$ & 0.009 \\
\hline LVEF drop $\geq 5 \%$ within the first $3 \mathrm{mo}$ & $9.29(4.21-20.49)$ & $<0.001$ & $28.91(12.06-69.33)$ & $<0.001$ \\
\hline
\end{tabular}

TRCD, trastuzumab-related cardiac dysfunction; HR, hazard ratio; CI, confidence interval; CAD, coronary artery disease; LVEF, left ventricular ejection fraction, DBP, diastolic blood pressure.

Table 4. Clinical manifestations and treatment of TRCD

\begin{tabular}{|c|c|c|c|}
\hline Variable & $\begin{array}{l}\text { Reversible TRCD } \\
\qquad(\mathrm{n}=44)\end{array}$ & $\begin{array}{l}\text { No reversible TRCD } \\
\qquad(\mathrm{n}=14)\end{array}$ & p-value \\
\hline Age, mean $\pm S D(y r)$ & $49.4 \pm 8.6$ & $49.7 \pm 7.3$ & 0.822 \\
\hline \multicolumn{4}{|l|}{ Symptoms at presentation } \\
\hline Asymptomatic & $30(68.2)$ & $10(71.4)$ & 0.600 \\
\hline Dyspnea & $11(25)$ & $4(28.6)$ & \\
\hline Palpitation & $3(6.8)$ & - & \\
\hline NYHA functional class II/III & $10(12.7)$ & $4(28.6)$ & 0.020 \\
\hline LVEF at diagnosis of TRCD, median (range) & $47.5(40.0-50.0)$ & $50.0(43.2-50.0)$ & 0.246 \\
\hline Discontinuation of trastuzumab & $22(50.0)$ & $5(35.7)$ & 0.351 \\
\hline Cardioprotective medications & $30(68.2)$ & $5(35.7)$ & 0.031 \\
\hline
\end{tabular}

Values are presented as number (\%) unless otherwise indicated. TRCD, trastuzumab-related cardiac dysfunction; NYHA, New York Heart Association; LVEF, left ventricular ejection fraction.

sive decline in LVEF during the first 3 months of trastuzumab treatment in patients who presented with TRCD compared with those without TRCD $(10.0 \%$ [5.0-16.0] vs. $2.0 \%$ [0-6.0], $\mathrm{p}<0.001)$. Within 3 months of treatment with trastuzumab, an LVEF drop of $5 \%$ or more was more frequently observed in patients with TRCD than those without TRCD (78.3\% vs. 38.4\%, p < 0.001) (Fig. 3). In multivariate analysis, low baseline LVEF, high baseline blood pressure, the use of high-dose Adriamycin and a drop in LVEF $\geq 5 \%$ within the first 3 months, were associated with the development of TRCD (Table 3).

\section{Treatment of TRCD}

Among 58 patients with TRCD, $60.3 \%$ initiated cardioprotective medication and $46.6 \%$ discontinued trastuzumab treatment. TRCD patients who discontinued trastuzumab and started cardioprotective medication improved LV systolic function after 4.5 months (range, 2.3 to 12.8 months). TRCD patients who continued the use of trastuzumab (31 patients, 53.4\%) had a higher LVEF at the time of TRCD diagnosis than those who discontinued trastuzumab $(50.0 \%$ [49.0-50.0] vs. 40.0\% [35.0-45.0], p < 0.001). LV dysfunction was completely improved in $75.9 \%$ of TRCD patients (Table 4 ). There was a higher rate of trastuzumab discontinuation in patients with improved TRCD compared with those whose TRCD did not improve, but it was not statistically significant (50.0\% vs. $35.7 \%, p=0.351)$. Patients with TRCD who had improved LVEF more frequently received cardioprotective medication regardless of the extent of LVEF decrement than those who did not have improved LVEF (68.2\% vs. $35.7 \%$, $\mathrm{p}=0.031$ ). The median duration of cardioprotective medica- 
tion was 19.0 (range, 11.0 to 26.0 months). During the median follow-up period (54.3 months), no trastuzumab-related cardiac deaths or heart transplantations occurred.

\section{Discussion}

In this observational study, we investigated clinical manifestations and predictors of TRCD in Asian patients who were treated with trastuzumab in the adjuvant setting for breast cancer. The overall incidence of TRCD was 7.4\%, including $2.3 \%$ of symptomatic heart failure. Our cohort showed a low prevalence of cardiac predisposing factors such as hypertension, diabetes, dyslipidemia, or coronary artery disease, and this was not different between the patients with or without TRCD. Before initiation of trastuzumab, low baseline LVEF and previous exposure to adriamycin were closely associated with the development of TRCD. During trastuzumab administration, LVEF dropped to $\geq 5 \%$ within 3 months regardless of baseline LV systolic function or whether adriamycin dose was an independent predictor of TRCD occurrence.

The incidence of TRCD is variable according to the age of the population and the presence of risk factors [2]. Previous clinical trials reported that the incidence of cardiac dysfunction after trastuzumab was much higher, ranging from $20 \%$ to $27 \%$, in subjects who were pre-treated with anthracycline $[16,17]$. In contrast, our data showed a relatively low incidence of TRCD despite the majority of patients previously receiving anthracycline. There are several possible explanations for our findings. First, the mean age of our cohort was 49.9 years, corresponding to previous data that showed an increment of young women with breast cancer in Asia [18]. Advanced age ( $>50$ years) is closely associated with the development of TRCD $[17,19,20]$; therefore, our results might be influenced by exposure to trastuzumab at a young age. The second explanation is that predisposing cardiac risk factors, including hypertension, diabetes, cerebrovascular accident, and coronary artery disease, were infrequent in our cohort. Therefore, a Western population might be predisposed to the baseline risk status before treatment with trastuzumab. Recently, Suter et al. [21] reported the incidence of congestive heart failure in patients who received anthracycline and / or trastuzumab in a large cohort. They also suggested that active monitoring for cardiac function during trastuzumab is critically important. However, they did not show specific data regarding the change in LVEF after trastuzumab, the exact number of patients who used trastuzumab and recovery of cardiac function. We thought that our data might be an evidence for suggesting a specific strategy for cardiac surveillance in breast cancer patients receiving trastuzumab by showing a precedent reduction in LVEF.

$\mathrm{LV}$ dysfunction during the use of trastuzumab has been defined as a decline in LVEF of at least $10 \%$ below normal or symptomatic heart failure according to protocols in previous clinical trials $[8,22]$. Regarding the interval of monitoring LV systolic function, current cardiology and oncology guidelines recommend serial examination of LVEF every 3 months during and once after completion of trastuzumab treatment in the adjuvant setting for patients with breast cancer. However, because these recommendations are based on expert opinion without additional supporting evidence, screening during trastuzumab treatment depends on local protocols and modality selection depends upon the local physicians and availability. Notably, in our analysis, we identified that a decline in LVEF of at least 5\% during the first 3 months regardless of baseline LVEF and anthracycline administration was strongly associated with TRCD. Furthermore, about $50 \%$ of TRCD patients had 'normal range of LVEF' at the first 3 months despite EF decreased more than 5\% than the baseline. Therefore, they continued administration of trastuzumab without any intervention. Considering the early detection of patients at risk of developing cardiac dysfunction during and after trastuzumab treatment is the most important parameter in reducing and reversing TRCD, recommendations regarding a strict threshold of LVEF decrement are needed. In this respect, outweighing number of patients with homogeneous baseline characteristics strengthens our results that emphasize the role of early serial changes of LVEF.

There is still debate about whether radiotherapy-induced cardiotoxicity and chemotherapy-induced cardiotoxicity are synergistic [23]. In contrast to the previous studies that report a high risk of cardiac mortality in patients who underwent breast radiotherapy in addition to trastuzumab [24,25], our data demonstrated no significant increases in adverse cardiac events in those who underwent radiotherapy during a median follow-up of 54.2 months. Our results might support those of recent clinical trials which showed an association between trastuzumab treatment, radiotherapy, and cardiotoxicity $[26,27]$.

Interestingly, there was less TRCD in obese patients in our data. The obesity paradox is phenomenon that has been proven in various studies related to cardiac disease. There have been several possible explanations for this phenomenon. First, many authors suggested clinical or neurohormonal aspects $[28,29]$. Production of adiponectin and anti-inflammatory cytokines such as tumor necrosis factor $\alpha$ (TNF- $\alpha$ ) receptor, and the ability to store glucose in obese patients are increased in medical or surgical critical illness [30,31]. We infer that in chemotherapy-related cardiotoxicity, a severely stressful situation, similar responses by adipose tissue or pericardial fat may function as cardio-protectors that decrease 
myocardial injury. It is possible that patients with overweight, but not with morbidly obesity, have relatively lower levels of harmful adipokines compared to lean patients because most participants had a body mass index $<30 \mathrm{~kg} / \mathrm{m}^{2}$ in this study. Although these have not been demonstrated in patients with chemotherapy-related cardiotoxicity, a study on blood levels of TNF- $\alpha$ receptor, adiponectin, and leptin in patients with myocardial damage due to other cause such as myocardial infarction might help clarify this presumption. Second explanation is that epicardial adipose tissue might contribute beneficially to coronary artery by fulfilling antiinflammatory, anti-oxidant, vasodilatory, and neuroprotective functions and may serve as a reservoir of free fatty acids for the energy needs of injured myocardium [32].

Our data showed that the patients with TRCD had less hypertensive, although it was not statistically significant. This might have affected the use of cardioprotective agents during anti-hypertensive medication. Patients with TRCD who initiated cardioprotective medications such as angiotensin-converting enzyme inhibitors, angiotensin receptor blockers, or $\beta$-blockers also showed a higher rate of LVEF recovery than those without cardioprotective medication. Yet, there have been no randomized trials supporting the finding that heart failure drugs prevent TRCD and improve cardiac function in patients with TRCD. Considering that the use of trastuzumab is indispensable for treatment of HER 2positive breast cancer, our data may provide evidence to establish monitoring protocols for the development of TRCD. Future prospective trials to evaluate the role of cardiac medication in reducing and restoring TRCD are warranted.

There were several limitations to the present study. First, this was a single-center observational study; thus, our results might be subject to unrecognized confounding factors. Although we performed multivariate analysis and adjusted for several potential confounding factors, we could not correct for unmeasured or unobserved variables. Second, we did not routinely perform myocardial strain, 3-dimensional echocardiography, or cardiac magnetic resonance imaging, which seem to better predict decreases in conventional LVEF, although the detection of subclinical changes in cardiac performance was more sensitive than standard echocardiography. Even though the above tools can detect cardiac dysfunction more accurately with high reproducibility, real-world clinical application of the tools was limited because of the high cost and time restrictions [33-35]. We believe that the use of two-dimensional echocardiography at an appropriate time interval remains a useful tool for the evaluation of chemotherapy-related cardiotoxicity [36]. Third, because most patients in this study were pre-treated with anthracycline, potential influence on the cardiac dysfunction after administration of trastuzumab cannot be excluded. However, we adjusted the influence of LV systolic function before trastuzumab and the use of anthracycline in multivariate analysis, therefore we believe that such confounders have little effect on the results. Lastly, we did not measure serum troponin levels, which can effectively indicate drug-induced cardiac injury in the early phase, long before any reduction in LVEF has occurred. However, we are now in the process of confirming the clinical relevance of such promising data with a prospective design.

In conclusion, the overall incidence of TRCD was $7.4 \%$ in Asian patients who were treated with adjuvant trastuzumab for breast cancer. Regardless of baseline LV systolic function and pre-treatment with Adriamycin, the drop in LVEF of $\geq 5 \%$ within the first 3 months after trastuzumab initiation strongly associated with TRCD development. Physicians should be particularly aware of small changes in LV systolic function in the early period of adjuvant trastuzumab treatment in breast cancer patients. Such monitoring could enable early detection of patients who are at risk of developing cardiac disease and in whom a cardioprotective approach to therapy is needed.

\section{Conflicts of Interest}

Conflict of interest relevant to this article was not reported.

\section{References}

1. Romond EH, Perez EA, Bryant J, Suman VJ, Geyer CE Jr, Davidson NE, et al. Trastuzumab plus adjuvant chemotherapy for operable HER2-positive breast cancer. N Engl J Med. 2005;353:1673-84.

2. Jones LW, Haykowsky MJ, Swartz JJ, Douglas PS, Mackey JR. Early breast cancer therapy and cardiovascular injury. J Am Coll Cardiol. 2007;50:1435-41.

3. Hahn VS, Lenihan DJ, Ky B. Cancer therapy-induced car- diotoxicity: basic mechanisms and potential cardioprotective therapies. J Am Heart Assoc. 2014;3:e000665.

4. Telli ML, Hunt SA, Carlson RW, Guardino AE. Trastuzumabrelated cardiotoxicity: calling into question the concept of reversibility. J Clin Oncol. 2007;25:3525-33.

5. Ewer SM, Ewer MS. Cardiotoxicity profile of trastuzumab. Drug Saf. 2008;31:459-67.

6. Wadhwa D, Fallah-Rad N, Grenier D, Krahn M, Fang T, 
Ahmadie R, et al. Trastuzumab mediated cardiotoxicity in the setting of adjuvant chemotherapy for breast cancer: a retrospective study. Breast Cancer Res Treat. 2009;117:357-64.

7. Tan-Chiu E, Yothers G, Romond E, Geyer CE Jr, Ewer M, Keefe D, et al. Assessment of cardiac dysfunction in a randomized trial comparing doxorubicin and cyclophosphamide followed by paclitaxel, with or without trastuzumab as adjuvant therapy in node-positive, human epidermal growth factor receptor 2-overexpressing breast cancer: NSABP B-31. J Clin Oncol. 2005;23:7811-9.

8. Zamorano JL, Lancellotti P, Rodriguez Munoz D, Aboyans V, Asteggiano R, Galderisi M, et al. 2016 ESC Position Paper on cancer treatments and cardiovascular toxicity developed under the auspices of the ESC Committee for Practice Guidelines: The Task Force for cancer treatments and cardiovascular toxicity of the European Society of Cardiology (ESC). Eur Heart J. 2016;37:2768-801.

9. Curigliano G, Cardinale D, Suter T, Plataniotis G, de Azambuja E, Sandri MT, et al. Cardiovascular toxicity induced by chemotherapy, targeted agents and radiotherapy: ESMO Clinical Practice Guidelines. Ann Oncol. 2012;23 Suppl 7:vii155-66.

10. de Azambuja E, Procter MJ, van Veldhuisen DJ, Agbor-Tarh D, Metzger-Filho O, Steinseifer J, et al. Trastuzumab-associated cardiac events at 8 years of median follow-up in the Herceptin Adjuvant trial (BIG 1-01). J Clin Oncol. 2014;32:2159-65.

11. Shah AM, Solomon SD. Myocardial deformation imaging: current status and future directions. Circulation. 2012;125:e244-8.

12. Romond EH, Jeong JH, Rastogi P, Swain SM, Geyer CE Jr, Ewer MS, et al. Seven-year follow-up assessment of cardiac function in NSABP B-31, a randomized trial comparing doxorubicin and cyclophosphamide followed by paclitaxel (ACP) with ACP plus trastuzumab as adjuvant therapy for patients with node-positive, human epidermal growth factor receptor 2-positive breast cancer. J Clin Oncol. 2012;30:3792-9.

13. Lang RM, Badano LP, Mor-Avi V, Afilalo J, Armstrong A, Ernande L, et al. Recommendations for cardiac chamber quantification by echocardiography in adults: an update from the American Society of Echocardiography and the European Association of Cardiovascular Imaging. J Am Soc Echocardiogr. 2015;28:1-39.e14.

14. Piccart-Gebhart MJ, Procter M, Leyland-Jones B, Goldhirsch A, Untch M, Smith I, et al. Trastuzumab after adjuvant chemotherapy in HER2-positive breast cancer. N Engl J Med. 2005;353:1659-72.

15. Plana JC, Galderisi M, Barac A, Ewer MS, Ky B, Scherrer-Crosbie $\mathrm{M}$, et al. Expert consensus for multimodality imaging evaluation of adult patients during and after cancer therapy: a report from the American Society of Echocardiography and the European Association of Cardiovascular Imaging. J Am Soc Echocardiogr. 2014;27:911-39.

16. McArthur HL, Chia S. Cardiotoxicity of trastuzumab in clinical practice. N Engl J Med. 2007;357:94-5.

17. Perez EA, Suman VJ, Davidson NE, Sledge GW, Kaufman PA, Hudis CA, et al. Cardiac safety analysis of doxorubicin and cyclophosphamide followed by paclitaxel with or without trastuzumab in the North Central Cancer Treatment Group
N9831 adjuvant breast cancer trial. J Clin Oncol. 2008;26: 1231-8.

18. Lin $\mathrm{CH}$, Shen $\mathrm{CY}$, Lee JH, Huang CS, Yang $\mathrm{CH}$, Kuo WH, et al. High prevalence of the BIM deletion polymorphism in young female breast cancer in an East Asian country. PLoS One. 2015;10:e0124908.

19. Seidman A, Hudis C, Pierri MK, Shak S, Paton V, Ashby M, et al. Cardiac dysfunction in the trastuzumab clinical trials experience. J Clin Oncol. 2002;20:1215-21.

20. Guglin M, Cutro R, Mishkin JD. Trastuzumab-induced cardiomyopathy. J Card Fail. 2008;14:437-44.

21. Suter TM, Procter M, van Veldhuisen DJ, Muscholl M, Bergh J, Carlomagno $\mathrm{C}$, et al. Trastuzumab-associated cardiac adverse effects in the herceptin adjuvant trial. J Clin Oncol. 2007;25:3859-65.

22. Choi JY, Cho EY, Choi YJ, Lee JH, Jung SP, Cho KR, et al. Incidence and risk factors for congestive heart failure in patients with early breast cancer who received anthracycline and/or trastuzumab: a big data analysis of the Korean Health Insurance Review and Assessment service database. Breast Cancer Res Treat. 2018;171:181-8.

23. Zagar TM, Cardinale DM, Marks LB. Breast cancer therapyassociated cardiovascular disease. Nat Rev Clin Oncol. 2016; 13:172-84.

24. Rutqvist LE, Lax I, Fornander T, Johansson H. Cardiovascular mortality in a randomized trial of adjuvant radiation therapy versus surgery alone in primary breast cancer. Int J Radiat Oncol Biol Phys. 1992;22:887-96.

25. Haybittle JL, Brinkley D, Houghton J, A'Hern RP, Baum M. Postoperative radiotherapy and late mortality: evidence from the Cancer Research Campaign trial for early breast cancer. BMJ. 1989;298:1611-4.

26. Halyard MY, Pisansky TM, Dueck AC, Suman V, Pierce L, Solin L, et al. Radiotherapy and adjuvant trastuzumab in operable breast cancer: tolerability and adverse event data from the NCCTG Phase III Trial N9831. J Clin Oncol. 2009;27:2638-44.

27. Zambelli A, Della Porta MG, Eleuteri E, De Giuli L, Catalano $\mathrm{O}$, Tondini $\mathrm{C}$, et al. Predicting and preventing cardiotoxicity in the era of breast cancer targeted therapies: novel molecular tools for clinical issues. Breast. 2011;20:176-83.

28. Angeras O, Albertsson P, Karason K, Ramunddal T, Matejka $\mathrm{G}$, James $\mathrm{S}$, et al. Evidence for obesity paradox in patients with acute coronary syndromes: a report from the Swedish Coronary Angiography and Angioplasty Registry. Eur Heart J. 2013;34:345-53.

29. Lavie CJ, Milani RV, Ventura HO. Impact of obesity on outcomes in myocardial infarction combating the "obesity paradox". J Am Coll Cardiol. 2011;58:2651-3.

30. Pingitore A, Di Bella G, Lombardi M, Iervasi G, Strata E, Aquaro GD, et al. The obesity paradox and myocardial infarct size. J Cardiovasc Med (Hagerstown). 2007;8:713-7.

31. Mohamed-Ali V, Goodrick S, Bulmer K, Holly JM, Yudkin JS, Coppack SW. Production of soluble tumor necrosis factor receptors by human subcutaneous adipose tissue in vivo. Am J Physiol. 1999;277:E971-5. 
32. Tamarappoo B, Dey D, Shmilovich H, Nakazato R, Gransar H, Cheng VY, et al. Increased pericardial fat volume measured from noncontrast CT predicts myocardial ischemia by SPECT. JACC Cardiovasc Imaging. 2010;3:1104-12.

33. Walker J, Bhullar N, Fallah-Rad N, Lytwyn M, Golian M, Fang $\mathrm{T}$, et al. Role of three-dimensional echocardiography in breast cancer: comparison with two-dimensional echocardiography, multiple-gated acquisition scans, and cardiac magnetic resonance imaging. J Clin Oncol. 2010;28:3429-36.

34. Sawaya H, Sebag IA, Plana JC, Januzzi JL, Ky B, Tan TC, et al. Assessment of echocardiography and biomarkers for the extended prediction of cardiotoxicity in patients treated with anthracyclines, taxanes, and trastuzumab. Circ Cardiovasc Imaging. 2012;5:596-603.

35. Thavendiranathan P, Poulin F, Lim KD, Plana JC, Woo A, Marwick TH. Use of myocardial strain imaging by echocardiography for the early detection of cardiotoxicity in patients during and after cancer chemotherapy: a systematic review. J Am Coll Cardiol. 2014;63:2751-68.

36. Villarraga HR, Herrmann J, Nkomo VT. Cardio-oncology: role of echocardiography. Prog Cardiovasc Dis. 2014;57:10-8. 